\title{
Keynote Symposium
}

\section{KS-1}

Seeing Single Molecules, from Early Spectroscopy in Solids, to Super-resolution Microscopy, to 3D Dynamics of Biomolecules in Cells. W. E. MOERNER. Stanford University, Department of Chemistry, Stanford, CA 94305. Email: wmoerner@stanford.edu

More than 25 years ago, low temperature experiments aimed at establishing the ultimate limits to optical storage in solids led to the first optical detection and spectroscopy of a single molecule in the condensed phase. At this unexplored ultimate limit, many surprises occurred where single molecules showed both spontaneous changes (blinking) and light-driven control of emission, properties that were also observed in 1997 at room temperature with single green fluorescent protein variants. In 2006, PALM and subsequent approaches showed that the optical diffraction limit of $\sim 200 \mathrm{~nm}$ can be circumvented with single molecules to achieve super-resolution fluorescence microscopy with relatively nonperturbative visible light. Essential to this is the combination of single-molecule fluorescence imaging with active control of the emitting concentration and sequential localization of single fluorophores decorating a structure. Super-resolution microscopy has opened up a new frontier in which biological structures and behavior can be observed in fixed and live cells with resolutions down to $20-40 \mathrm{~nm}$ and below. Examples range from protein superstructures in bacteria to details of the shapes of amyloid fibrils and much more. Current methods development research addresses ways to extract more information from each single molecule such as 3D position and orientation, and both of these can be obtained by proper point-spread function engineering of a wide-field microscope. It is worth noting that in spite of all the current focus on super-resolution, even in the "conventional" low concentration, single-molecule tracking regime where the motions of individual biomolecules are recorded rather than the shapes of extended structures, much can still be learned about biological processes. Examples to be presented include studies of the primary cilium and motions of DNA loci in the nucleus.

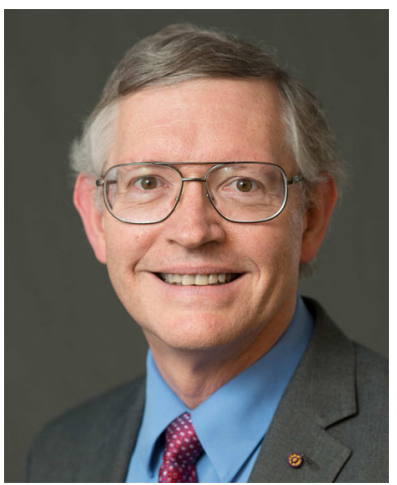

